\title{
Simulation numérique de l'Estuaire de la Loire
}

\author{
Rosales-Sierra Victor $^{\mathrm{a}}$, Levacher Daniel ${ }^{\mathrm{b}}$
}

\author{
${ }^{a}$ Docteur, ${ }^{b}$ Professeur, Université de Caen, UMR 6143 CNRS Morphodynamique \\ Continentale et Côtière, 24 rue des Tilleuls, 14000 Caen, France, Téléphone: +33 \\ 231565709, Fax: +33231565709 \\ E-mail : victor.rosales@free,fr \\ daniel.levacher@geos.unicaen.fr
}

\section{Résumé}

Un ensemble de résultats relatifs à la simulation numérique de l'Estuaire de la Loire est présenté. La méthode habituelle de comparaison avec des mesures n'est pas toujours précise pour valider des simulations numériques, en particulier, pour des cas d'études d'une complexité importante. Dans le cas de la Loire, le manque de données et la complexité des codes de calcul empêchent toute reproduction acceptable en modèle numérique de la physique de l'Estuaire.

Pour approfondir l'étude sur la Loire, il faudrait un système de mesures permanent qui permette de connaître l'historique des paramètres au long de l'Estuaire recueilli à intervalles réguliers en conditions normales et, irréguliers pour les événements extraordinaires. Les systèmes de calcul comme Telemac3D seront d'autant plus performants si une routine de vérification et de validation est automatique pour connaître l'incertitude numérique et pour déceler plus facilement des erreurs.

Des conclusions de cette étude donnent une idée de l'espacement du maillage et de l'intervalle de temps dans le modèle. La comparaison de la convergence des différents maillages permet d'observer le niveau de raffinement du modèle selon le type de simulation. Pour une simulation relative à l'hydrodynamique, on peut utiliser des maillages grossiers alors que pour une simulation liée à la salinité on doit utiliser des maillages plus raffinés. Quant à la simulation des sédiments en suspension, le code n'est pas encore capable de donner des résultats satisfaisants au niveau de la convergence.

\section{Introduction}

Lors d'une étude d'ingénierie avec simulation numérique, l'ingénieur est confronté au choix des paramètres qui génèrent divers résultats. Le choix se fait souvent par calage des résultats de la simulation avec des mesures. Une fois le modèle calibré de cette façon, des prédictions avec différents scénarii sont envisageables. Pour chaque scénario, les conditions aux limites et les paramètres changent. Les résultats sont validés en supposant que l'incertitude de la simulation de calage est la même pour toutes les simulations.

Il est démontré dans cet article que cette hypothèse n'est pas fondée dans tous les cas. En particulier, ceux dont la complexité de l'étude est importante.

Dans le cas de l'Estuaire de la Loire, la complexité des phénomènes en présence dans l'Estuaire et les limites du modèle utilisé amplifient les erreurs au niveau des résultats. Pour cette raison, le calage avec des mesures s'avère insuffisant pour valider les résultats numériques.

LE NORMANT $(1995)^{1}$ et CHEVIET et al. $(2000)^{2}$ ont utilisé le code TELEMAC3D $\left(\right.$ JANIN, 1992) ${ }^{3}$ pour effectuer des simulations de l'Estuaire de la Loire avec succès en utilisant un seul et en calant les résultats avec des mesures faites sur site (APEEL, 1993) ${ }^{4}$. 
Dans ce travail, la méthode de vérification et de validation décrite par ROSALES et al. $(2002)^{5}$ a été appliquée pour valider des résultats de trois maillages raffinés.

Cette étude a permis de repérer une divergence dans le code de calcul et de la corriger. Malgré cette amélioration, le manque de données empêche de poursuivre en détails l'étude.

\section{Le modèle numérique de la Loire}

On a utilisé la géométrie de l'estuaire proposée par LE HIR $(1994)^{6}$ pour une simulation numérique. Notons aussi qu'un estuaire schématique est plus adapté aux besoins de la recherche pour mettre en évidence les erreurs de divergence de codes tridimensionnels à surface libre. La géométrie ainsi retenue permet de négliger les effets de bord de l'Estuaire et simplifie la propagation de la marée.


Figure 1 Comparaison de profils mesurés et modélisés

Bien que le fond bathymétrique mesuré soit similaire dans le modèle numérique, l'aire hydraulique n'est pas tout à fait en accord avec la section moyenne de l'Estuaire. Ceci implique que l'aire hydraulique est très proche de la réalité quand seulement le niveau de la mer est proche du zéro. La

Figure 1 montre deux profils de l'Estuaire de la Loire à différents points kilométriques.

Cette simplification est en partie due au manque d'informations et de mesures relatives aux bords de l'Estuaire. De ce fait une reproduction plus fidèle des bords génère plus d'incertitudes, et qui plus est, on ne peut faire de calages.

En ce qui concerne le domaine de calcul, illustré par les Figures 2 et 3, il représente 106 $\mathrm{km}$ de longueur pour une largeur variable dont la moyenne est de $600 \mathrm{~m}$. La bathymétrie est représentative du chenal de navigation de la Loire selon les données établies en 1987. Le plan horizontal, Figure 3, a été réparti en 805 nœuds, c'est-à-dire 1280 éléments triangulaires pour le maillage en éléments finis. 




Figure 2 Domaine de calcul du modèle de la Loire (coupe longitudinale, axe du chenal)

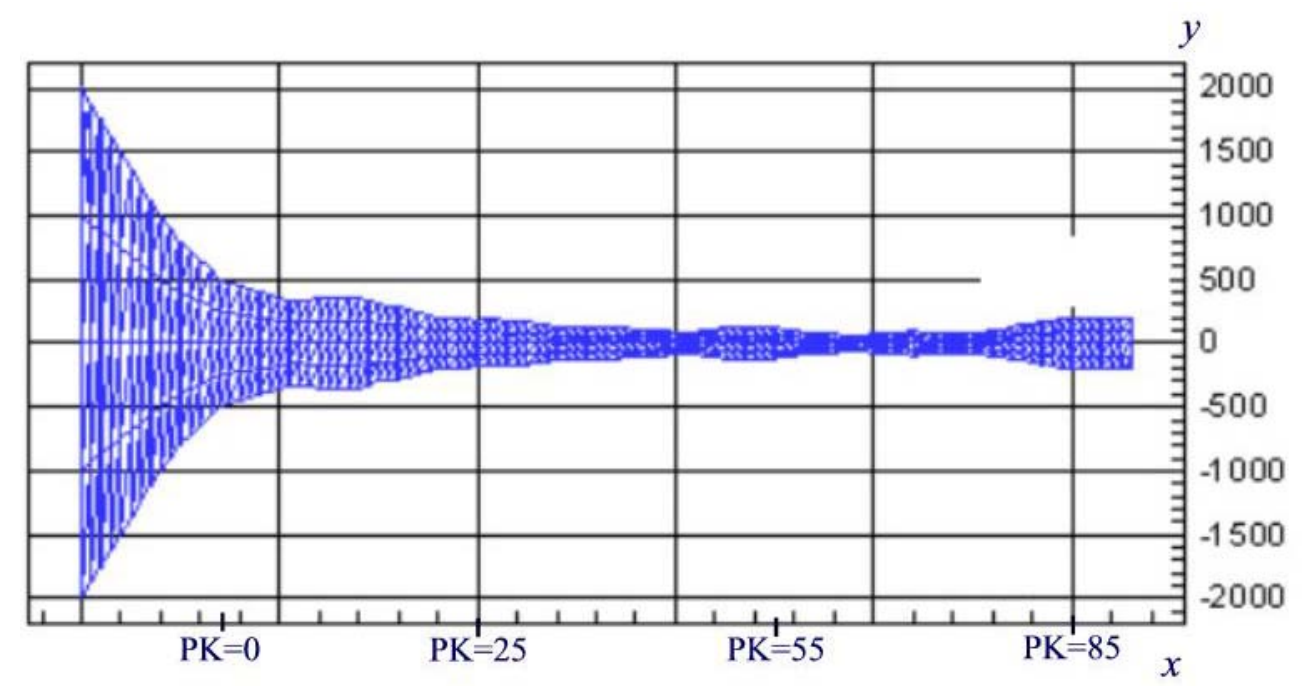

Figure 3 Domaine de calcul du modèle de la Loire (vue en plan)

La répartition des mailles dans les modèles, a été faite de tel manière que l'axe du chenal de navigation coïncide avec l'axe $0 y$. Suivant la direction $x$, on conserve un espacement $x$ constant, égal à $1 \mathrm{~km}$. Dans la direction $y$, l'espacement est variable ; on divise la largeur de l'estuaire en 4 parties d'égale dimension. On adopte aussi dans la direction $z$, un espacement variable. On divise ainsi la profondeur en 16 couches d'épaisseur constante. L'espacement suivant la direction $z$ a de plus, la particularité de pouvoir évoluer en fonction des oscillations de la surface libre.

Le point initial du domaine de calcul est décalé de $15 \mathrm{~km}$ en aval de Saint-Nazaire $(\mathrm{PK}=0)$. La condition limite maritime est donc éloignée pour éviter l'influence _brusque du fond sur la propagation de l'onde de marée dans le modèle numérique. La bathymétrie est lissée dans certains endroits pour éviter des inconsistances numériques à cause des variations soudaines du fond. A l'amont, la bathymétrie a été simplifiée à l'aide d'une pente régulière moins prononcée. 
Dans la pratique, on cale les résultats en les confrontant aux mesures de terrain. Pour cela, on modifie la valeur du coefficient de frottement et de diffusion visqueuse. Cette manipulation permet de filtrer les faites à la géométrie pour s’approcher des mesures.

\section{Analyse des mesures de terrain}

Les deux forçages les plus importantes dans la dynamique estuarienne sont la marée et le débit fluvial. L'hydrodynamique, la pénétration saline et la concentration en sédiments sont fonction de la combinaison de ces deux forçages avec la géomorphologie de l'estuaire.

La marée est de type semi-diurne avec une période de $12 \mathrm{~h} 24 \mathrm{mn}$ environ. L'amplitude de la marée varie périodiquement entre un coefficient $C_{m}$ de 95 ( $5 \mathrm{~m}$ de marnage) et un coefficient de 45 (2,3m de marnage) pendant 14,5 jours. Le débit moyen de la Loire est de $825 \mathrm{~m}^{3} / \mathrm{s}$. Le débit de crue varie entre 2000 et $5000 \mathrm{~m}^{3} / \mathrm{s}$ alors que celui d'étiage évolue entre 100 et $250 \mathrm{~m}^{3} / \mathrm{s}$ (mesures extrêmes décennales à la station de Montjean). Un autre phénomène d'intérêt est la stratification. Elle est présente près de l'embouchure lors d'un débit fluvial de creu $\left(+2000 \mathrm{~m}^{3} / \mathrm{s}\right)$ et une marée assez faible. La stratification provoque une intrusion de la salinité par le fond.

Pour la simulation numérique, on simplifie les données relatifs aux mesures pour établir un cas général. Par exemple, on simule des conditions de faible marnage avec un débit fluvial de creu pour analyser la stratification ou des conditions d'étiage avec un fort marnage pour analyser la pénétration saline dans l'Estuaire. Un des problèmes réside dans le fait qu'il y a un manque de mesures suffisantes pour bien reproduire l'un ou l'autre cas. La situation idéale serait d'avoir un historique de tous les paramètres étudiés en différents points de l'Estuaire pour bien caler le modèle. De ce fait, les résultats obtenus sont difficilement comparables avec la réalité. Cependant, on effectue un calage du modèle avec les rares mesures disponibles de l'Estuaire. Pour s'approcher de ces mesures, dans la pratique on surestime les coefficients numériques de viscosité augmentant ainsi le risque de divergence numérique.

Dans le cas de notre étude, on a tout d'abord ressemblé des mesures de niveau en plusieurs points de l'Estuaire et de salinité en deux points dans la même période. La manipulation des coefficients de frottement et de diffusion visqueuse permet de s'approcher des valeurs mesurées. Pour une étude classique, cela suffirait pour valider le modèle. 

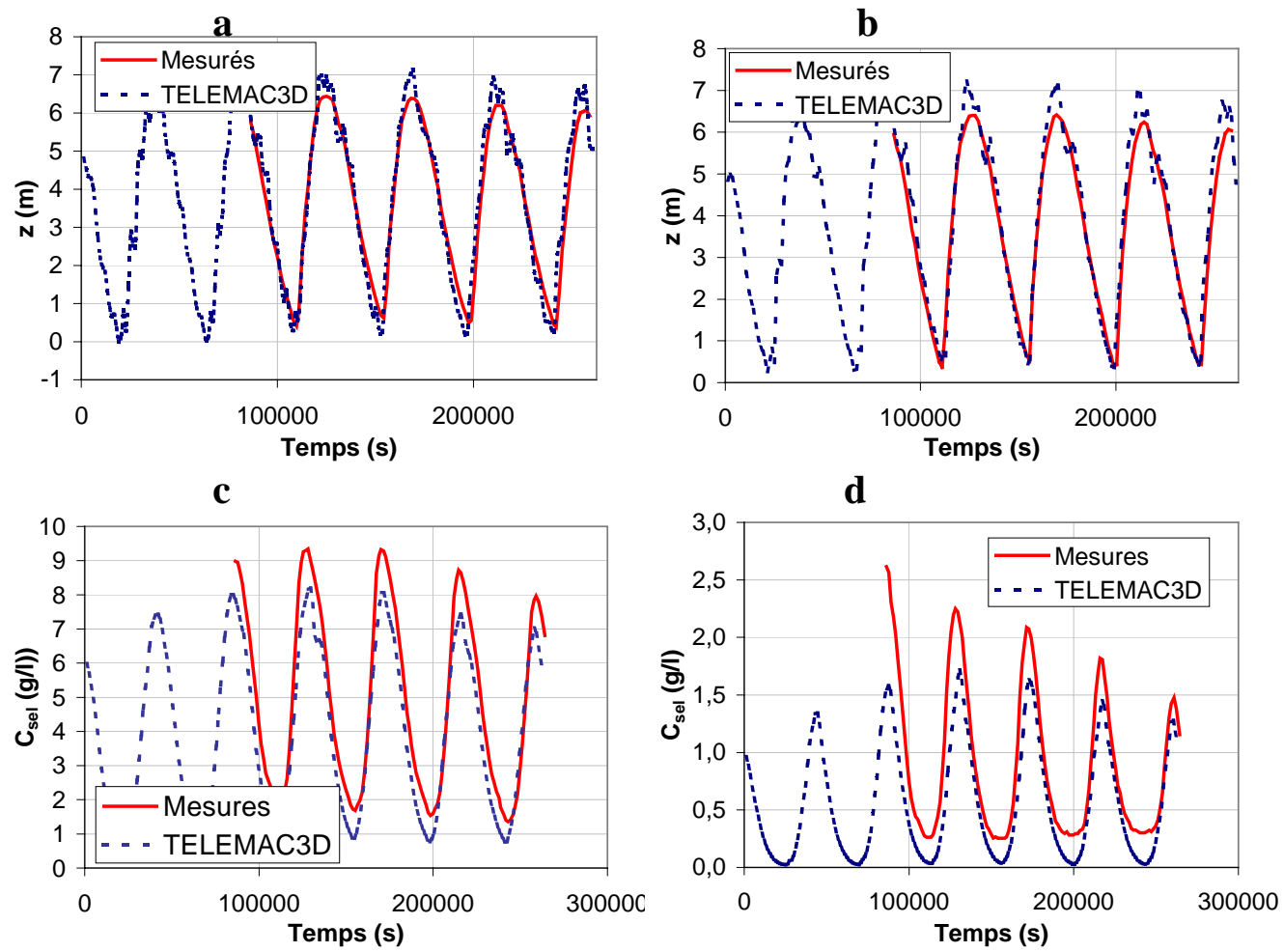

Figure 4 Calage du modèle avec des mesures sur site de niveaux d'eau (z) au $P K=38$ (a), au $P K=55$ (b) et de salinité $\left(C_{s e l}\right)$ au $P K=38$ (c), au $P K=55$ (d)

La Figure 4 montre les résultats numériques avec les mesures de terrain.

\section{Analyse de convergence du code de simulation}

Le code utilisé est destiné à la modélisation des écoulements à surface libre en site maritime ou fluvial. Il résout les équations tridimensionnelles de Navier-Stokes moyennes par Reynolds et l'équation de transport d'un traceur. Ce traceur est actif s'il agit sur la densité de l'eau et donc sur l'écoulement. Il est passif s'il n'agit pas sur l'écoulement et dans ce cas, il est uniquement transporté. Cet outil permet donc de traiter en particulier des études de dilution thermique, de pollution et de transport sédimentaire. Il est donc très performant dans le cas de l'Estuaire de la Loire.

Pour avoir plus de rigueur sur la validation du code on a utilisé la méthode de vérification et validation décrite par ROSALES et al. (2002). Cette méthode utilise trois maillages raffinés distincts. La validation est fait en cas de convergence des résultats : les solutions des mailles plus raffinés tendent vers une valeur déterminée. Dans le cas contraire il y a divergence. Lors de premiers résultats on a observé des inconsistances numériques dans des maillages raffinés (divergence), même si le calage était correcte. 
Par souci de commodité et de sophistication, on a divisé l'étude en trois phases. Une première phase concerne l'hydrodynamique. La seconde à rajouter la salinité. Puis finalement, dans la dernière phase la concentration en sédiments a été introduite. La divergence des solutions a été observée dans cette dernière phase. On a ainsi constaté que dans les études précédents CHEVIET (2000) et LE NORMANT (1995), cette erreur n'a pas été remarquée probablement à cause de l'utilisation de coefficients de diffusion visqueuse très élevés qui agissent comme des filtres numériques dans le cas d'un maillage grossier.


Figure 5 Profils de concentration en sédiments avec et sans correction

La Figure 5 montre en détails une comparaison de profils de concentration en sédiments (Csed) au $\mathrm{PK}=28000$. On constate que sur la solution corrigée, le profil correspondant au maillage grossier (en pointillé) est clairement éloigné des profils correspondants aux maillages moyen (en trait) et fin (continu). La correction a été opérée sur le calcul de la vitesse de cisaillement au fond qui permet de mettre en suspension les sédiments. La divergence n'est repérable qu'en comparant les résultats des trois maillages.

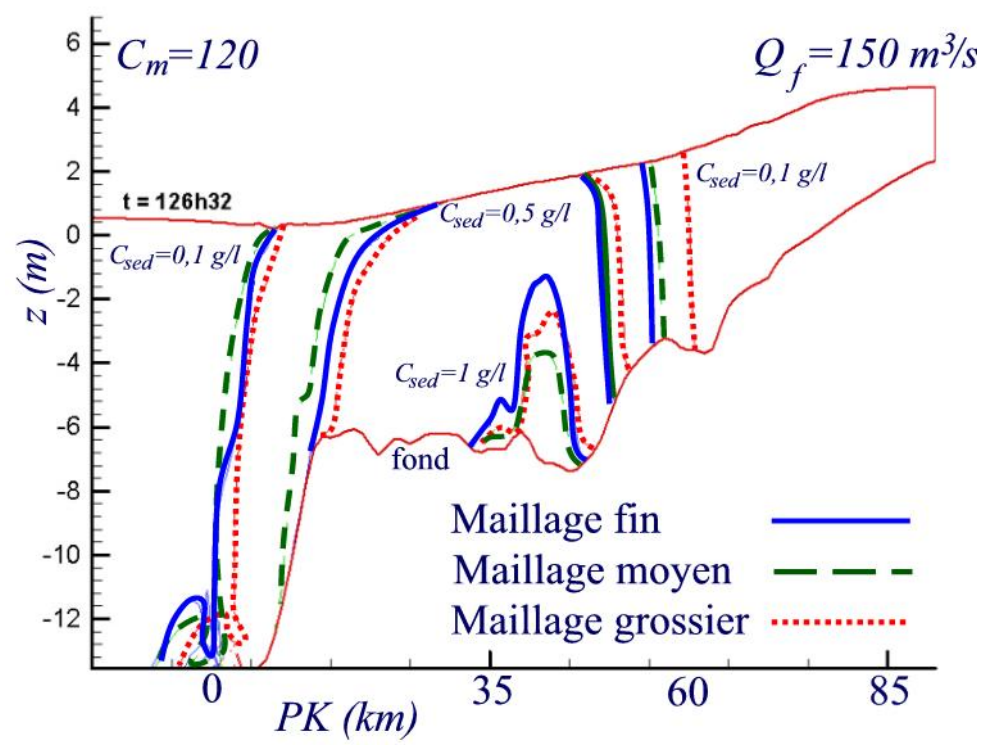

Figure 6 Comparaison de résultats relatifs à la concentration en sédiments pour les trois maillages utilisés 
Malheureusement, la correction n'est pas encore parfaite. En comparant les résultats des trois maillages dans tout le domaine sur la Figure 6, on constate encore une divergence des solutions. La divergence s'affirme plus claire dans la zone de l'embouchure. Cela peut s'expliquer si l'on considère les simplifications faites sur les conditions aux limites.

\begin{tabular}{|c|c|c|c|}
\hline Maillage & $\begin{array}{c}\text { Espacement } \\
\text { de maille, } \\
\text { direction } x\end{array}$ & $\begin{array}{c}\text { Plans } \\
\text { quasi- } \\
\text { horizontaux }\end{array}$ & $\begin{array}{c}\text { Pas } \\
\text { de } \\
\text { temps }\end{array}$ \\
\hline Fin & 500 & 34 & 30 \\
\hline Moyen & 662.5 & 24 & 40 \\
\hline Grossier & 1000 & 17 & 60 \\
\hline
\end{tabular}

Tableau 1 Caractéristiques des maillages

Malheureusement, le manque de données empêche d'autres confrontations des résultats mais à ce stade au moins, on est en mesure de vérifier le code et d'envisager des développements futurs.

A partir des simulations et de l'analyse de vérification, on peut recommander un ordre de grandeur de l'espacement du maillage et de l'intervalle de temps dans le modèle. La comparaison de la convergence des différents maillages a permis d'observer le niveau de raffinement du modèle selon le type de simulation. Pour une simulation de l'hydrodynamique, on peut utiliser un maillage grossier, pour une simulation liée à la salinité, il faut un maillage fin. Quant à la simulation des sédiments en suspension, le code n'est pas encore capable de donner des résultats satisfaisants au niveau de la convergence.

\section{Conclusions}

On constate avec cette étude que la physique de l'Estuaire de la Loire est encore loin de pouvoir être reproduite. En effet, des obstacles à tout développement concret concernent par l'essentiel la pénurie des données de terrain et les limites et performances du code de calcul.

Tout d'abord, on a besoin d'un système de mesures dans l'Estuaire de la Loire, une sorte d'observatoire permanent. En différents points kilométriques des mesures de niveau de l'eau, de vitesse, de direction des courants, de salinité et de concentration en sédiments à différentes profondeurs à intervalle régulier dans le temps devraient être mesurées. Des profils du fleuve doivent aussi être établis pour suivre notamment l'évolution des bords par rapport à la physique de l'Estuaire.

D'ailleurs, une automatisation de la méthode de vérification et de validation dans Telemac3D est nécessaire. En effet, même s'un travail important a été fait sur l'automatisation du calage, il faudra ajouter une forme d'auto vérification avec les trois maillages. Ceci permettra d'une part de repérer des erreurs dans le code et, d'autre part, d'estimer le degré d'incertitudes dans les études. 


\section{Références}

1.Le Normant C., (1995). Modélisation numérique tridimensionnelle des processus de transport des sédiments cohésifs en environnement estuarienne. Thèse de Doctorat, Institut Polytechnique de Toulouse, France, 236 p.

2.Cheviet C., Violeau D., Guesmia M., (2000). MAST-3 COSINUS European project 3DModelling of cohesive sediment transport in the Loire estuary (France), EDF-DER, France, 38 p.

3.Janin J. M., Lepeintre F., Pechon P., (1992). Telemac-3D : A finite element code to solve 3D free surface flow problems. Computer modeling of seas and coastal regions, Southampton, (UK), $18 \mathrm{p}$.

4.APEEL, PANSN., (1993) Bilan de l'hydrologie et de l'hydrosédimentaire de l'estuaire de la Loire, aux cours des dernières décennies, Synthèse, Association pour la protection de l'environnement de l'estuaire de la Loire et Port Autonome de Nantes - Saint Nazaire, France, $53 \mathrm{p}$

5.Rosales V., Levacher D., Garapon A., (2002). Simulation numérique d'un écoulement stratifié turbulent, VII journées nationales Génie Civil - Génie Côtier, Anglet, France, 15-17 mai 2002, 105-115

6.Le Hir P., (1994). Fluid and sediment integrated modelling application of fluid mud flows in estuaries, in Cohesive sediments, proceedings of INTERCOH 1994, 417-428 